\title{
Calcium Channel Blockers: New Therapeutic Targets for Alzheimer's Disease
}

\section{Hooman Bozorgi*, Hamed Darbanian}

Department of Pharmacology, Research Center of Physiology and Neurosciences, School of Medicine, Semnan University of Medical

\author{
Sciences, Semnan, Iran
}

\section{Article Info:}

\section{A BSTRACT}

Introduction: Alzheimer's disease (AD) is the most common type of dementia in the elderly. Memory impairment usually occurs gradually and progressively. AD has not only been associated with decreased cerebral acetylcholine concentration, oxidative stress, and beta-amyloid protein deposition, but more recent studies have shown that neurodegeneration induced by disturbance of the intracellular calcium homeostasis is also involved in the pathophysiology of the disease. It has been reported that calcium channel blockers (CCBs) have beneficial effects in different models of memory impairment. Here, we review and discuss the effects of CCBs, especially N-types, as novel agents that have effects on memory deficits in animal models of AD. on contrary to the L-type calcium channels, which are presented in the other organs in addition to nerve cells, N-type calcium channels are commonly located on the neurons. The main advantage of N-type CCBs is their lower side effects than other CCBs. Conclusion: Targeting the N-type CCBs may contribute to the new strategies for the treatment of $\mathrm{AD}$.

*Corresponding Author: Hooman Bozorgi

E-mail: hoomanbozorgi@semums.ac.ir 


\title{
مسدود كنندههاى كانال كلسيمى: اهداف جديد درمانى بيمارى آلزايمر
}

\author{
هومن بزركى"، حامد دربانيان \\ كروه فارماكولوزى، مركز تحقيقات فيزيولوزى و علوم اعصاب، دانشكده يزشكى، دانشعاه علوم يزشكى سمنان، سمنان، ايران
}

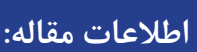

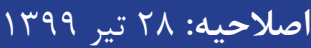

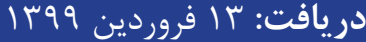

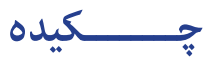

مقدمه: بيمارى آلزايمر شايعتر ين نوع زوال عقل دمانس در سالخورد كان محسوب مى شود. اختلال حافظه

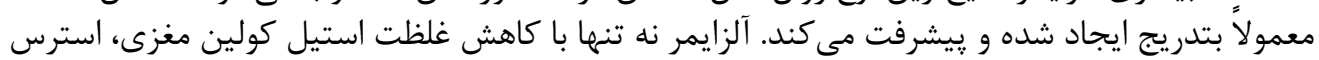

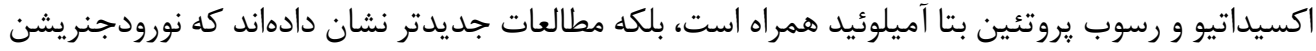

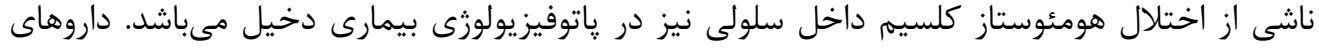

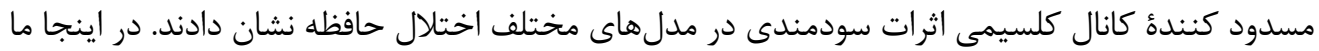

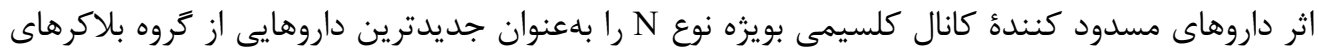

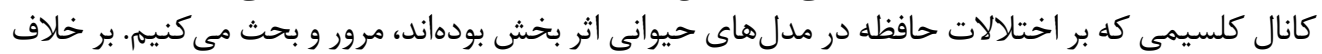

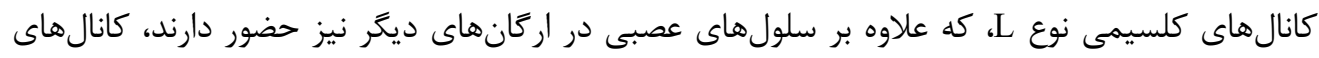

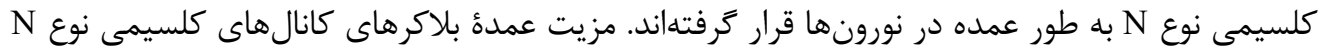

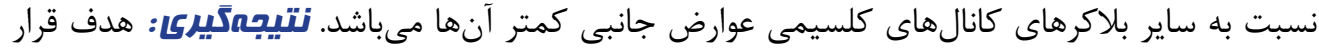

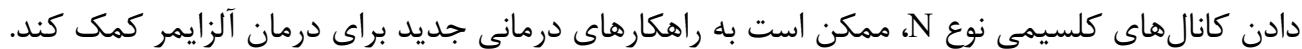

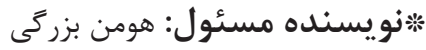

بـ بست الكترونيك: hoomanbozorgi@semums.ac.ir 


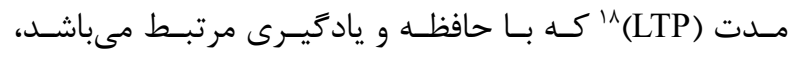

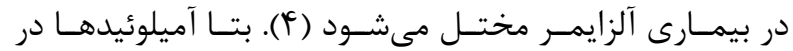

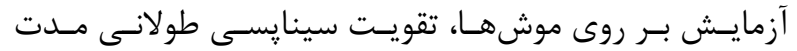

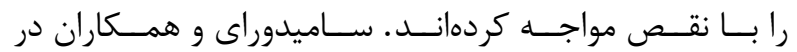

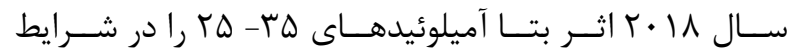

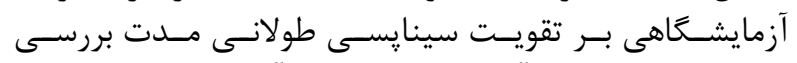

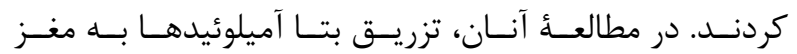

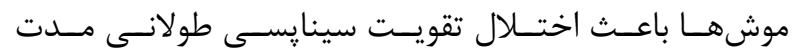

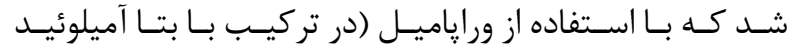

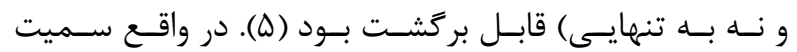

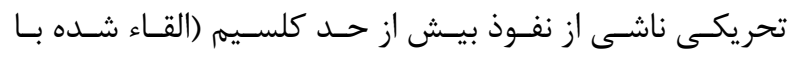

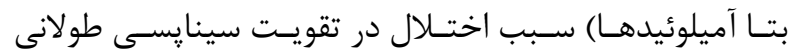

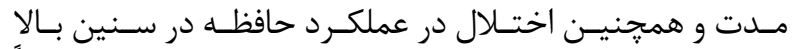

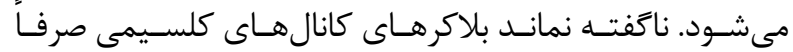

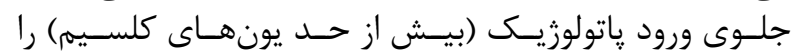

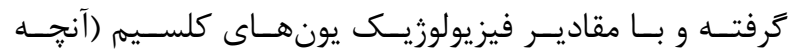

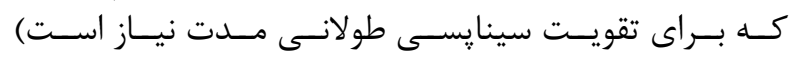

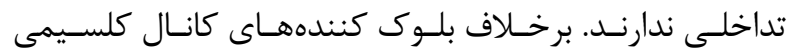

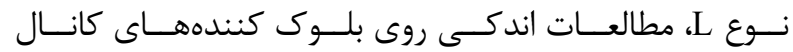

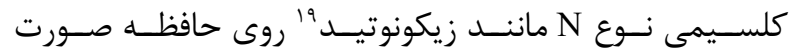

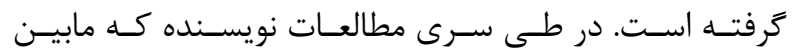

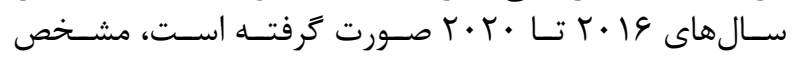

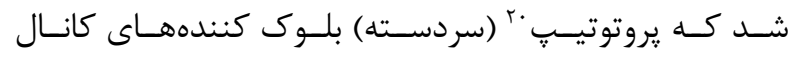

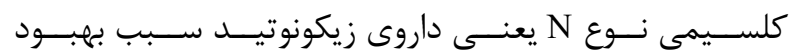

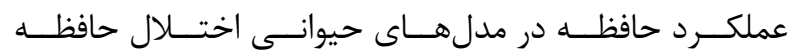

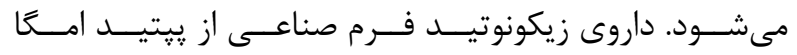

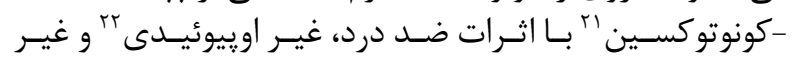

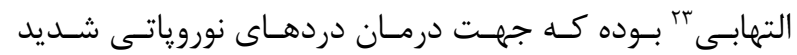

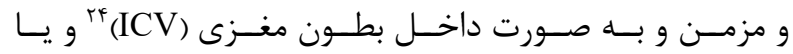

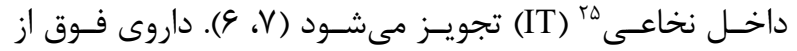

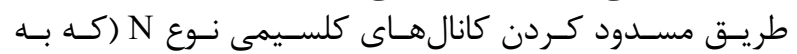

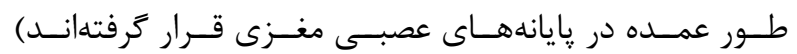

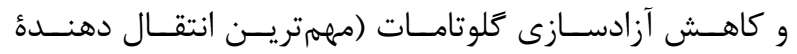

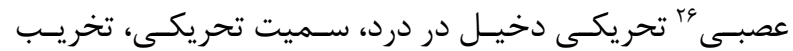

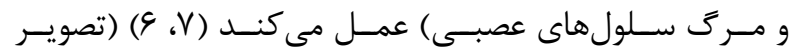

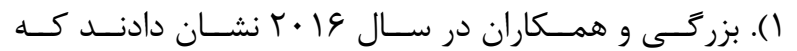

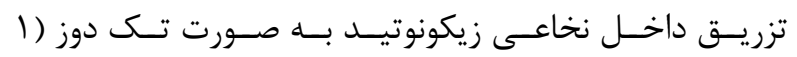

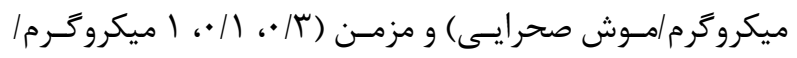

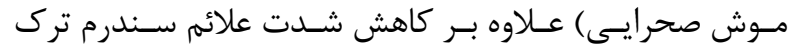

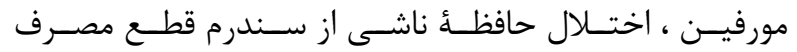

\section{${ }^{1}$ Excitotoxicity}

${ }^{2}$ Degeneration

${ }^{3}$ Aging

${ }^{4}$ Beta amyloid

${ }^{5}$ Presenilin gene

${ }^{6}$ Endoplasmic reticulum

${ }^{7}$ Glutamate

${ }^{8}$ N-Methyl D-Aspartate

${ }^{9}$ Hippocampus

${ }^{10}$ Anterior cortex

${ }^{11}$ Alzheimer's disease

${ }^{12}$ Dementia

${ }^{13}$ Neuroprotection

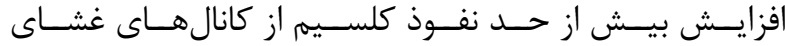

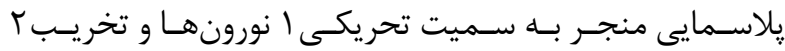

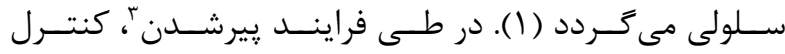

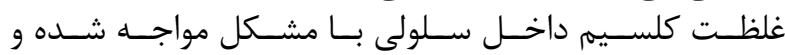

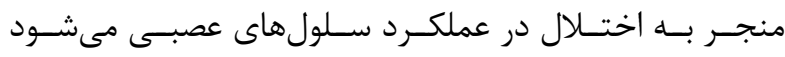

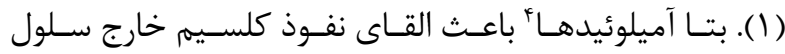

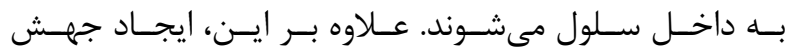

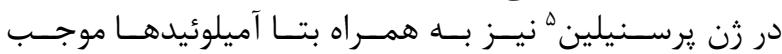

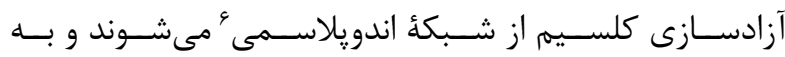

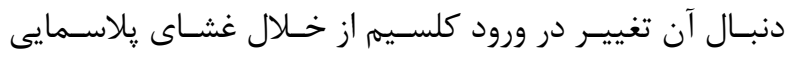

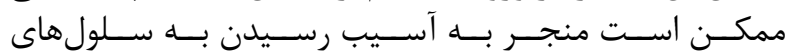

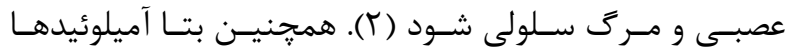

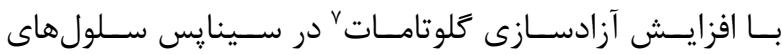

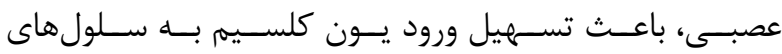

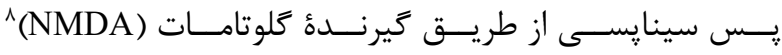

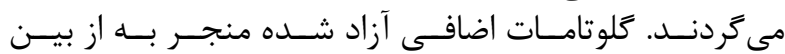

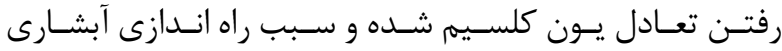

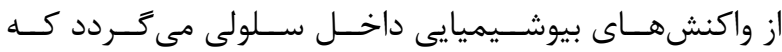

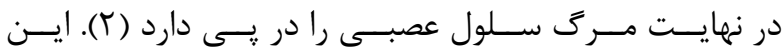

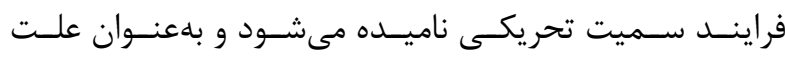

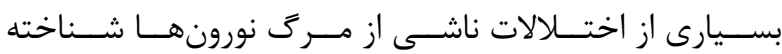

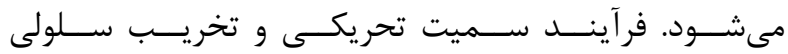

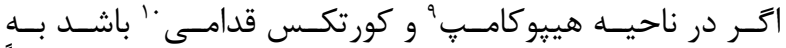

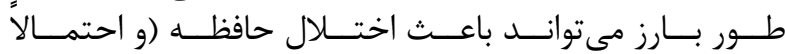

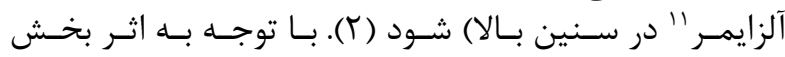

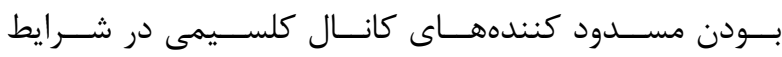

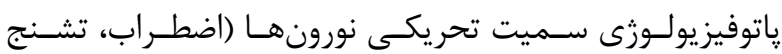

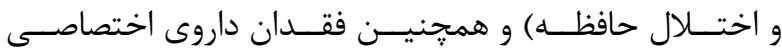

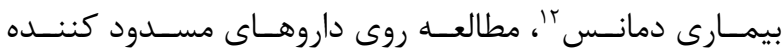

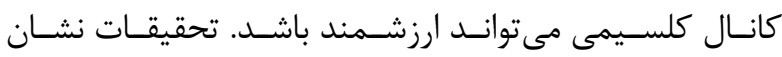

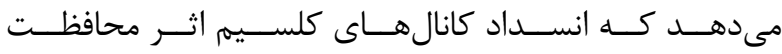
نورونسى" (عصبـى) را بـهـ دنبــال دارد (ب ، ()).

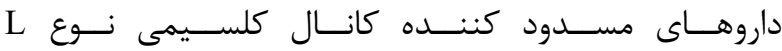

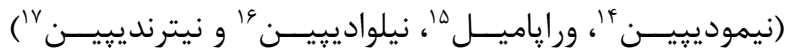

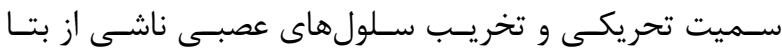

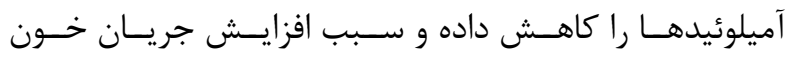

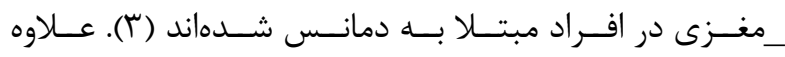

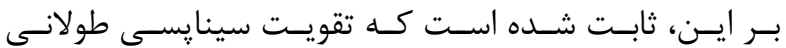

\footnotetext{
${ }^{14}$ Nimodipine

${ }^{15}$ Verapamil

${ }^{16}$ Nilvadipine

${ }^{17}$ Nitrendipine

${ }^{18}$ Long term potentiation

${ }^{19}$ Ziconotide

${ }^{20}$ Prototype

${ }^{21}$ Omega conotoxin

${ }^{22}$ Non opioid

${ }^{23}$ Non inflammatory

${ }^{24}$ Intra cerebro ventricular

${ }^{25}$ Intrathecal

${ }^{26}$ Neurotransmitter
} 


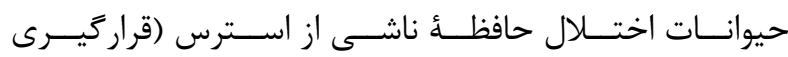

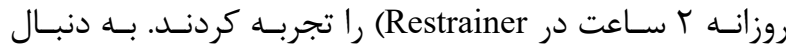

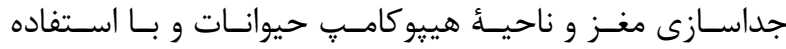

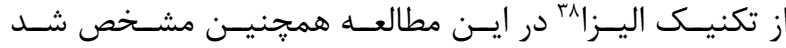

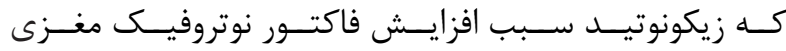

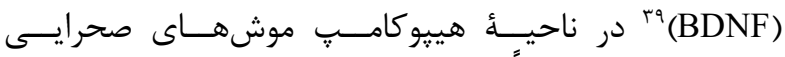

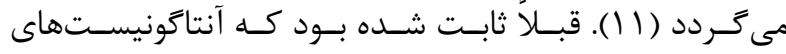

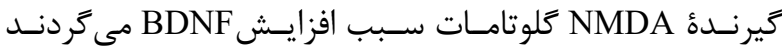

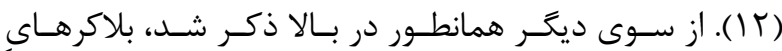

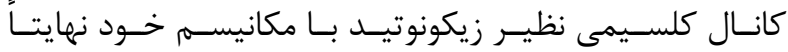

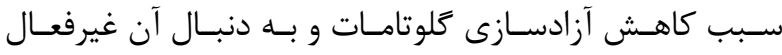

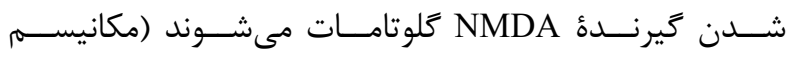

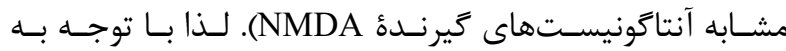

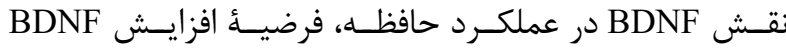

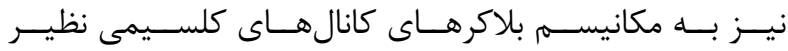

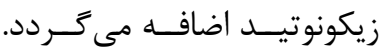

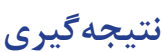

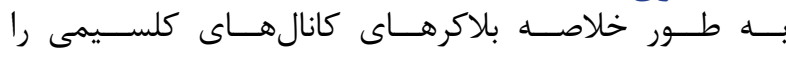

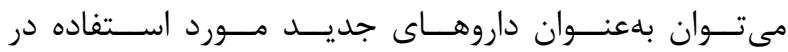

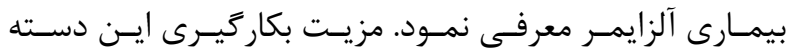

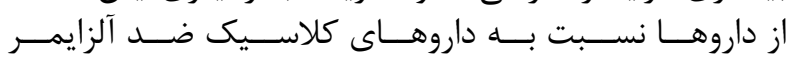

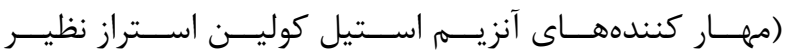

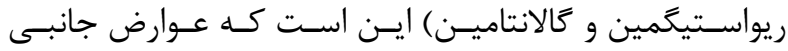

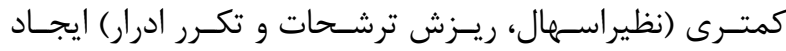

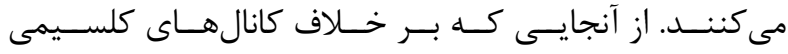

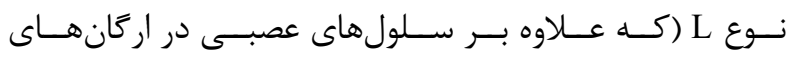

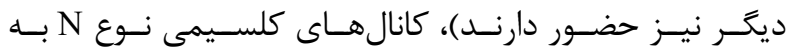

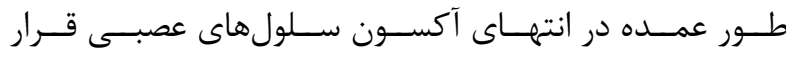

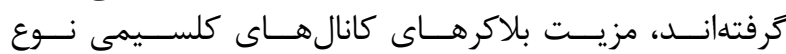

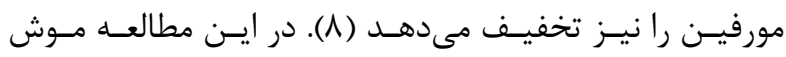

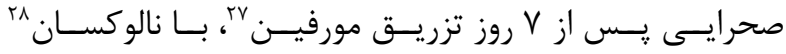

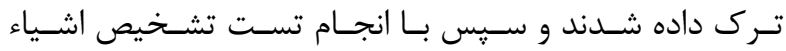

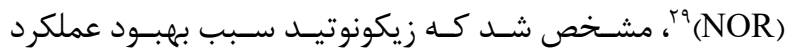

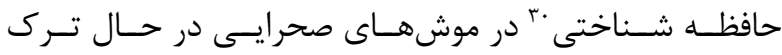

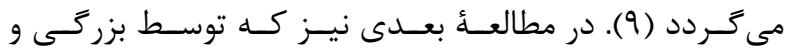

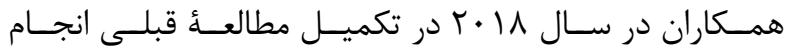

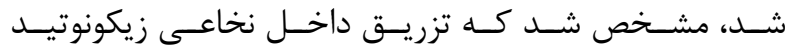

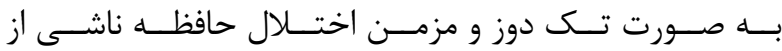

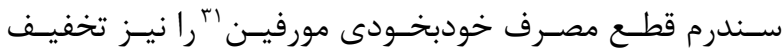

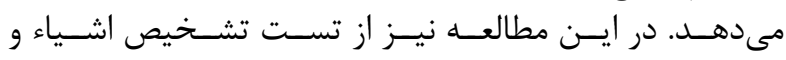

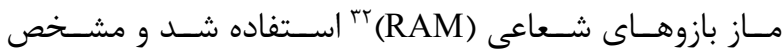

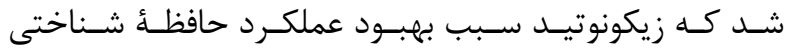

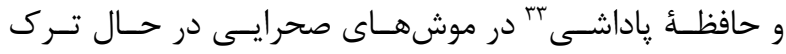

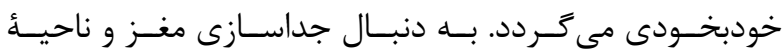

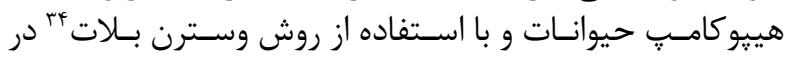

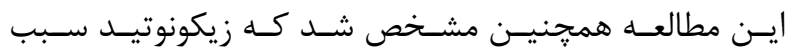

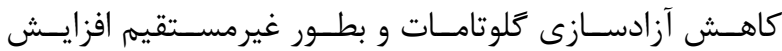

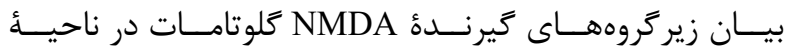

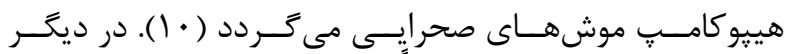

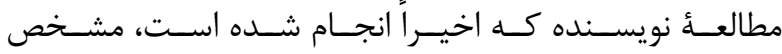

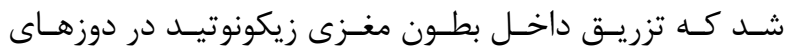

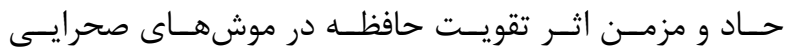

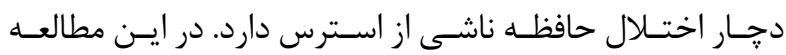

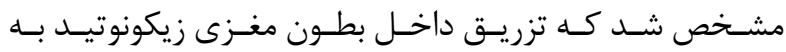

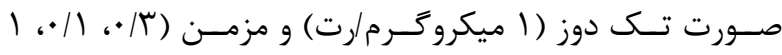

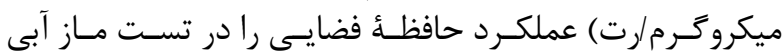
(MWM)

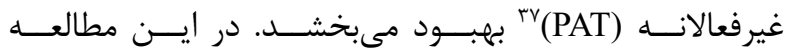

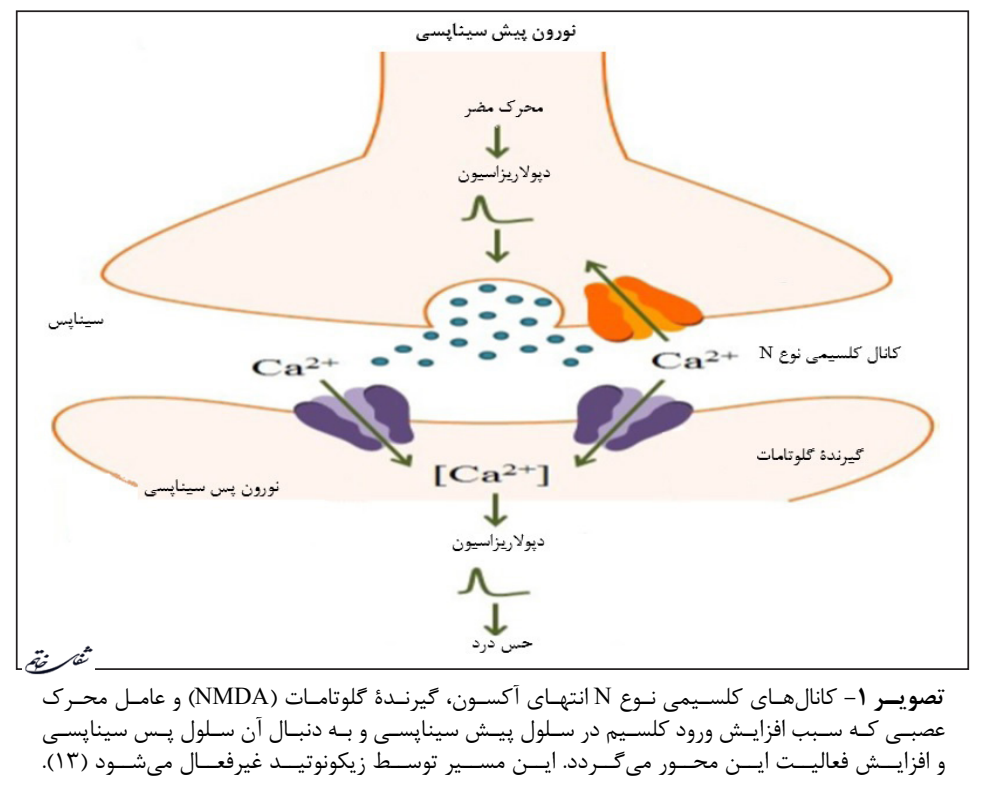

${ }^{27}$ Morphine

${ }^{28}$ Naloxone

${ }^{29}$ Novel object recognition

${ }^{30}$ Cognitive memory

${ }^{31}$ Spontaneous morphine withdrawal

${ }^{32}$ Radial arm maze

${ }^{33}$ Reward memory

\author{
${ }^{34}$ Western blot \\ ${ }^{35}$ Morris water maze \\ ${ }^{36}$ Conditional fear memory \\ ${ }^{37}$ Passive avoidance test \\ ${ }^{38}$ ELISA technique \\ ${ }^{39}$ Brain-derived neurotrophic factor
}




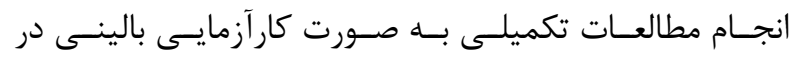

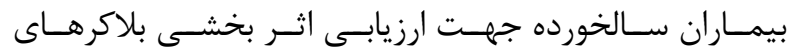

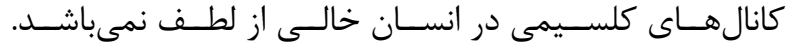

1. Feuerstein G, Hunter J, Barone FC. Calcium channel blockers and neuroprotection. Emerg Strateg Neuroprotection. 2013; 129-50.

2. Nimmrich V, Eckert A. Calcium channel blockers and dementia. Br J Pharmacol. 2013; 169: 1203-10.

3. Chiozzi P, Sarti AC, Sanz JM, Giuliani AL, Adinolfi E, Vultaggio-Poma V, et al. Amyloid $\beta$-dependent mitochondrial toxicity in mouse microglia requires $\mathrm{P} 2 \mathrm{X} 7$ receptor expression and is prevented by nimodipine. Sci Rep. 2019; 9: 6475.

4. Heale R. Dementia care and treatment issues. Evid Based Nurs. 2020; 23: 40-2.

5. Freir DB, Costello DA, Herron CE. A $\beta 25$-35-induced depression of long-term potentiation in area CA1 in vivo and in vitro is attenuated by verapamil. J Neurophysiol. 2003; 89: 3061-9.

6. Schmidtko A, Lötsch J, Freynhagen R. Ziconotide for treatment of severe chronic pain. Lancet. 2010; 375: 1569-77.

7. Skov MJ, Beck JC, de Kater AW. Nonclinical safety of ziconotide: an intrathecal analgesic of a new pharmaceutical class. Int J Toxicol. 2007; 26: 411-21.

8. Bozorgi H, Jahanian-Najafabadi A, Rabbani. The effect of intrathecal administration of the neuronal N-type calcium channels antagonist, $\omega$-conotoxin

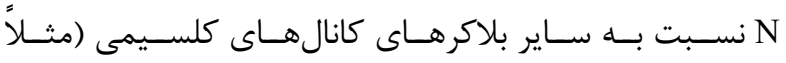

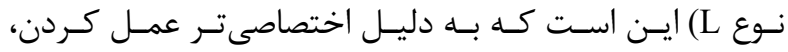

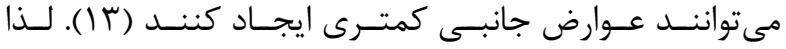

منابع

MVIIA, on attenuating the spontaneous and naloxoneprecipitated morphine withdrawal in rats. Toxin Rev. 2016; 35: 33-7.

9. Bozorgi H, Jahanian-Najafabadi A, Rabbani M. Effect of N-type calcium channel blocker Ziconotide on memory in rats following morphine withdrawal. Online J Vet Res. 2016; 20: 335-44.

10. Bozorgi H, Motaghi E, Zamani M, Ghavimi R. Neuronal calcium channels blocker, ziconotide ( induced memory impairments via alteration in hippocampal NMDA receptor expression in rats. Toxin Rev. 2018; 38: 1-10.

11. Bozorgi H, Budde T, Nankali M. Antidepressant-like and memory-enhancing effects of the N-type calcium channel blocker, ziconotide in rats. Behav Brain Res. 2020; 390.

12. Deutschenbaur L, Beck J, Kiyhankhadiv A, Mühlhauser M, Borgwardt S, Walter M, et al. Role of calcium, glutamate and NMDA in major depression and therapeutic application. Prog Neuropsychopharmacol Biol Psychiatry. 2016; 64: 325-33.

13. Hannon HE, Atchison WD. Omega-Conotoxins as Experimental Tools and Therapeutics in Pain Management. Mar Drugs. 2013; 11: 680-99. 